\title{
Ethnobotany of medicinal plants in the dayak limbai tribe
}

\section{Markus Iyus Supiandi (D*, Yakobus Bustami, Benediktus Ege, Hendrikus Julung, F. R. Esti} Wahyuni, Jul Afrianus

Biology Education Study Program, STKIP Persada Khatulistiwa Sintang

"Corresponding author: msupiandi@gmail.com

Article Info
Article History:
Received 28 Agust 202I
Revised $\quad$ I9 September 202I
Accepted $\quad$ I8 October 202I
Published 30 November 202I

Keywords:

Ethnobotany

Plants

Medicine

Traditional

Dayak limbai
ABSTRACT

The use of medicinal plants by the Dayak Limbai tribe has been going on for a long time, it's just that knowledge related to the use of plants as medicinal ingredients has not been well documented. The aims of this study are I) to identify plant species that have the potential as medicinal ingredients; 2) describe the part of the medicinal plant used; 3) describe how to use plants as medicine by the Dayak Limbai people. This research was conducted with a qualitative descriptive approach. The data collected consisted of the diversity of medicinal plants (local names of medicinal plants, scientific names of medicinal plants, medicinal plant families, parts used, and their utilization). Data collection using structured interview sheets. Data analysis using content analysis. The results obtained as many as 82 types of plants used to treat 64 types of diseases. The most widely used plant species by the Dayak Limbai people are from the Zingiberaceae family.

Copyright (C) 202I, Supiandi et al This is an open access article under the CC-BY-SA license

Citation: Supiandi, M. Bustami, Y., Ege, B., Julung, H., Wahyuni, F.R.E., \& Afrianus, J. (202I). Ethnobotany of medicinal plants in the dayak limbai tribe. JPBIO (Jurnal Pendidikan Biologi), 6(2), 244-26I. DOI: https://doi.org/I0.31932/jpbio.v6i2.1382

\section{INTRODUCTION}

The Dayak tribe is one of the indigenous tribes of Kalimantan. The Dayak have different customs and traditions from each other. Dayak is a common designation in Kalimantan, which indicates one of the tribes in Indonesia that inhabit the island of Kalimantan. Geographically and populations generally live along watersheds (Hamidah, Rijanta \& Marfai, 20I4). The existence of the Dayak tribe is divided into 405 sub-tribes, one of which is the Dayak Limbai tribe (Riwut, 1993). Administratively the Dayak Limbai people are in Batas Nangka Village, Menukung Subdistrict, Melawi Regency has traditional knowledge in using medicinal plants to treat various types of diseases.

Medicinal plants are defined as a type of plant in which part or all of the plant in the form of roots, stems, leaves, flowers, fruits, and seeds are used as medicine, ingredients, or medicinal 
herbs (Herbie, 2015). Medicinal plants are the use of biodiversity around us, both cultivated plants and wild plants. Medicinal plants have a relationship with traditional medicine because most of the utilization of medicinal plants is based more on the experience of use by some people who are already qualified (Harmida, Sarno \& Yuni, 20II). Knowledge and use of medicinal plants are only limited to knowledge in terms of the processing, use, and efficacy of plants (Haryono, Wardenaar \& Yusro, 20I4). The utilization of traditional medicinal plants for the maintenance of health and disease disorders is still needed (Efremila, Wardenaar \& Sicily, 20I5).

Dayak Limbai people use plants as traditional medicine for several reasons, among others: a) plants have the potential to be very much in the forest, b) plants are used as an alternative to healing if there are no health workers, c) processing can be done simply and does not require costs. These reasons are also supported by Lestari, Jamhari \& Isnainar (2017) which state that the reason a community group uses plants as traditional medicine is that it has the potential of adequate natural resources and human resources.

Knowledge in the Dayak Limbai tribe about the use of medicinal plants to treat various diseases is threatened with extinction due to several factors, namely: a) knowledge of medicine is only controlled by the elderly, b) young people today are less motivated to explore knowledge from the elderly, c) have entered palm oil companies and forests are cut down so that the types of plants are reduced, d) fields move in various forests, e) the high cost of medicines. Conditions like this over time will cause traditional knowledge in utilizing plants as medicine to experience extinction in the original place (Noorcahyani, 2012; Efremila et al., 20I5). One way to reduce the threat of extinction to knowledge about utilizing plants as a medicine is to document through ethnobotany study of medicinal plants.

Ethnobotany is a study that studies the relationship of human culture to the plant-based nature around it without damaging or exploiting it (Artha, 2016). Ethnobotany science revolves around the use of plants by people around them, in its application can increase human life (Kandowangko, 20II). Ethnobotany to protect the intellectual property of local people in the form of knowledge of the use of plants as medicine to avoid the possibility of exploitation, not only physical objects but also documentation (Munawaroh, 20I2).

Ethnobotany science is important to develop because with ethnobotany research, an understanding of the success or fallacy of traditional societies in understanding their environment, to avoid the same mistakes in the present or the future (Walujo, 20II). Ethnobotany science must also be applied in the student environment, one of which is as a support for botany courses. Ethnobotany understanding is also expected to open students' insights about the importance of local knowledge so that it needs to be preserved and expected while increasing students' love for local wisdom in the community (Silalahi, 2020). The study of botany is not only about botany, but also concerns traditional ethnobotany knowledge owned by local people (Dharmono, 2007). The purpose of this study is to gather information from the Dayak Limbai people about how to use plant organs as traditionally processed drugs.

\section{RESEARCH METHODS}

\section{Research Design}

This study uses a qualitative descriptive approach that describes the data as-is and by the facts found in the Dayak Limbai tribe about the use of plants as medicine. Qualitative descriptive research is one of the types of research included in the type of qualitative research (Moleong, 2010). The purpose of this study is to reveal the events or facts, phenomena, and circumstances that occur during the study by presenting what happened. This study interprets and deciphers data related to the situation that is happening in society by existing conditions (Nazir, 20I I) 


\section{Respondent}

Respondents to this study are people who in their daily lives use plants as medicinal ingredients and people who know medicinal plants. The technique of selecting respondents using purposive sampling. The purposive sampling technique was chosen because it suits the needs of research, namely the respondents selected must be indigenous people of the Dayak Limbai tribe and used to use plants for medicine. Some of the respondents selected in this study consisted of I customary administrator, I traditional medicine, 4 people who had an understanding of the use of medicinal plants.

\section{Instruments}

The instrument used in this study was a structured interview sheet. A structured interview is a type of interview where the question to be asked has been made before and becomes a guideline for the questions asked during the interview. The interview was addressed to indigenous administrators, traditional medicine, and Dayak Limbai people who know the traditional use of medicinal plants.

\section{Procedures} table I.

The research procedure conducted by the researcher consists of several stages presented in

Table I. The research procedure

\begin{tabular}{|c|c|c|c|}
\hline No & Time & Stages of Activity & Activity Details \\
\hline $\mathrm{I}$ & February 202I & Compile an interview sheet & $\begin{array}{l}\text { The interview sheet is arranged in the } \\
\text { form of a table that contains the local } \\
\text { name of the plant, the name of the } \\
\text { disease, the part used, how it is used. }\end{array}$ \\
\hline 2 & March 30, 202I & Research licensing & $\begin{array}{l}\text { Researchers delivered a research } \\
\text { license to the Head of Batas Nangka } \\
\text { Village. }\end{array}$ \\
\hline 3 & $\begin{array}{l}\text { March 3I, 202I - } \\
\text { April 5, 202I }\end{array}$ & Interview & $\begin{array}{l}\text { Researchers conducted interviews } \\
\text { with } 6 \text { respondents consisting of I } \\
\text { customary administrator, I traditional } \\
\text { medicine, and } 4 \text { people who have an } \\
\text { understanding of the use of medicinal } \\
\text { plants. }\end{array}$ \\
\hline 4 & $6-7$ April 202I & $\begin{array}{l}\text { Photo taken of medicinal } \\
\text { plants }\end{array}$ & $\begin{array}{l}\text { Researchers assisted by traditional } \\
\text { medicine take photos of plants in the } \\
\text { forest. }\end{array}$ \\
\hline 5 & May 202I & Analysis of research data & $\begin{array}{l}\text { Researchers determine the scientific } \\
\text { name of medicinal plants obtained } \\
\text { from the field. }\end{array}$ \\
\hline
\end{tabular}

\section{Data Analysis}

Data analysis techniques in this study in the form of qualitative descriptive by analyzing ethnobotany of medicinal plants using content analysis based on data that has been obtained. The interview data will be grouped by plant type (local name, scientific name, family), disease name, part of the plant used, and how it is used.

\section{RESULTS}


The results of this study were obtained through interviews and field observations with respondents in the tribe community of Dayak Limbai. The results showed different numbers for the number of plant species used as medicine by the tribe of Dayak Limbai community, consist of 6 species from the Zingiberaceae family, 4 species from the Euphorbiaceae family, 4 species from the Moraceae family, 4 species from the Poaceae (Gramineae) family, 3 species from the Lamiaceae family, each 2 species from several families which include Solanaceae, Musaceae, Anacardiaceae, Malvaceae, Myrtaceae, Cucurbitaceae, Piperaceae, Alliaceae, Rubiaceae, Arecaceae, Fabaceae, and Acoraceae. For several other families including Blechnaceae, Schizaeaceae, Umbiliferae, Araceae, Iridaceae, Clusiaceae, Amaryllidaceae, Phyllanthaceae, Athyriaceae, Oxalidaceae, Caricaceae, Convolvulaceae, Simaroubaceae, Leguminosae, Melastomataceae, Amaranthaceae, Nephrolepidaceae, Louraceae, Melied Polyaceae, Louraceae, Menispermaceae, Dipterocarpaceae, Annonaceae, Dilleniaceae, Crassulaceae, Marantaceac, Rutaceae, and Lycopodiaceae each consisted of only I species used. There is I species that has not been identified. The results of the study are presented on the appendix I.

The tribe community of Dayak Limbai people use plants as medicine in I8 ways, namely by pounding, dicing, boiling, smearing, eating, drinking, scraping, sticking, burning, cutting, heated over a fire, eaten raw, eaten directly, woven, wrapped, chewed, sprayed, and dripped. Of the I8 ways of utilization, the most widely used method is by smearing, because the type of disease being treated requires treatment outside the body. For treatment in the body, it is still done by eating (after vegetables or boiling), drinking (after boiling or making drinks), eaten raw (without vegetables or boiled or burned), eaten directly (usually because the fruit is ripe from the tree). The parts of plants used for medicine include rhizomes, leaves, roots, fruit, sap, bark, shoots, dry leaves, young leaves, stems, flowers, tubers, seeds, stem pulp, buds, bulbs (tuber layers), water in leaf shoots, dried fruit, old corn silk, fruit skin, old fruit, ripe fruit, young flowers, young stems, fruit juice, and stem shoots. Of the 26 plant parts that can be used for medicine, the most widely used is the leaf, which can be young or old.

\section{DISCUSSION}

Based on interviews and field observations with respondents in the Dayak Limbai tribe, Menukung Subdistrict, Melawi Regency obtained as many as 82 types of plants used to treat 64 types of diseases. The number of medicinal plants found in the Dayak Limbai tribe because the area still has a large enough forest so that the forest has the potential as a place or habitat for medicinal plants used by the local community. This is in line with what was conveyed by Anisah (202I) that forests in Indonesia (including the Dayak Limbai tribe) are known as mega biodiversity and as producers of plants that have medicinal ingredients. Furthermore, Simamora (2018) stated that forests are natural resources that have many benefits, among others, beneficial for ecology, socio-culture, economy, and produce non-timber for medicine.

Dayak Limbai people use parts (organs) of plants namely roots, rhizomes, stems, skin, leaves, flowers, fruit, seeds, sap, and eye buds to perform the treatment of various types of diseases traditionally. The use of these plant parts or organs is due to the following reasons: (a) the knowledge is obtained from dreams, (b) the knowledge is conveyed for generations, (c) the parts of the plant are easily acquired and the availability in the forest is very much, (d) the harvesting of the plant's organs for treatment will not result in the plant dying. These reasons are also supported by the results of research Julung, Supiandi, Ege, Mahanal \& Zubaidah (2018); Supiandi \& Leliavia (2020) who reported that the use of medicinal plant parts was obtained from ancestral messages and Bala Petara through dreams and passed down from ancestors or parents.

In theory or the results of previous research explained various reasons why society uses the roots, rhizomes, stems, skin, leaves, flowers, fruit, seeds, sap, and eye buds. As for the detailed 
description as reported by Supiandi, Julung, Ege, Mahanal, \& Zubaidah (2020) that the Dayak people utilize the root part for traditional medicine because it is believed that the root part has high efficacy and efficacy in curing disease when accompanied by certain spells. Supriadi (2020) mentioned that rhizomes have antioxidant content for disease prevention and health care. According to Sofiah (2014) reported that the stem has the function to transport water and food substances and become a stockpiling of food substances so that the stem contains many substances that are good for the body. According to Sofiah (2014), the utilization of stem skin is usually used for the treatment of external diseases.

Oktavia, Darma \& Sujarwo (2017) mentioned that the leaf part is also easy to obtain, easy to extract, and is a place of accumulation of photosynthetic that can cure diseases. Furthermore, Lestaridewi, Jamhari \& Isnainar (2017) mentions that the leaves have a soft structure so that it is easy to process. Pardede (2013) mentions the use of fruit as a drug because chemically the fruit contains water, carbohydrates, proteins, vitamins, and minerals needed by the body. According to Sofiah (2014) reported that seeds have substances that are beneficial to the body. According to Rahayu, Sunarti, Sulistriani \& Prawiroatmodjo (2006) mentions the utilization of plant sap aims so that the content of compounds in the material can be maintained. According to Adfa (2005) that in general the active compounds found in the plant (roots, rhizomes, stems, skin, leaves, flowers, fruits, seeds) in the form of secondary metabolites such as alkaloids, flavonoids, terpenoids, steroids, coumarins, which have effects as antiviral, anti-cancer, anti-inflammatory, antioxidant, anti hepatoxic, and anti-diabetic.

Dayak Limbai people use plants as traditional medicine by pounding and smearing, boiled and drunk, taped, chewed and sprayed, cooked and eaten, burned and smeared, chewed and wrapped. These methods are simple and still very traditional. This is done by the community for several reasons, namely: (a) it does not require expensive costs or even does not require costs, (b) the process of doing or concocting does not take a long time, (c) simply use simple equipment so that anyone can do it. These reasons are also supported by the results of relevant research conducted by Adyana (2012); Supiandi, Zubaidah, Mahanal, Julung \& Ege (2019) mentioned that the majority of Dayak tribe processing easily and simply.

In theory or the results of previous research explained various reasons why a concocting medicinal plants by boiling and drinking, pounded and applied, taped, chewed and sprayed, cooked and eaten, burned and smeared, chewed and wrapped. As for the description in detail as reported by Supiandi et al (2020); Uzlifah (2014) reported that processing by boiling and drinking will produce preparations containing active substances and able to increase antioxidant activity. Efremila et al (2015) mention the processing of plants by pounding and smearing mostly to treat external diseases and will give and reaction when applied to the sick part. Nurhaida, Usman \& Tavita (2015) reported that the use of plants medicine by taping, the disease they feel will heal and have a reaction so quickly. Shah, Usman \& Yusro (20I4) mentions processing by cooking and eating mostly to treat internal diseases, plants that have been cooked and eaten will have a good impact on healing. Wulandari, Fitmawati \& Sofiyanti (20I4) reported that processing by burning and smearing to treat external diseases, the processing is seen from the part of plant organs used, to take saris and active substances contained in the plant has more benefits for healing. Depdikbud (I99I) reported processing by chewed and sprayed used to treat diseases that are not visible, and also sometimes to treat pain due to physical statehood. Depdikbud (I99I) mentions the processing of treatment by dressing mostly to treat diseases that are visible from the physical outside and only wrapped in the sick part.

\section{CONCLUSION}


Plants are used as medicine by the Dayak Limbai tribe as many as 84 types of plants are used to treat 64 types of diseases. The parts used to treat various diseases start from the roots, rhizomes, stems, skin, leaves, flowers, fruit, seeds, sap, and eye buds and are still done simply. The number of medicinal plants found in the Dayak Limbai tribe because the area still has a large enough forest so that the forest has the potential as a place or habitat for medicinal plants used by the local community.

\section{ACKNOWLEDGMENT}

Thanks were conveyed to the Chairman of Perkumpulan Badan Pendidikan Karya Bangsa Sintang, Chairman of STKIP Persada Khatulistiwa Sintang, respondents, field assistants who have helped this entire series of research.

\section{REFERENCES}

Adfa, M. (2005). Survey Etnobotani, Studi Senyawa Flavonoid dan Uji Brine Shrimp Beberapa Tumbuhan Obat Tradisional Suku Serawai di Propinsi Bengkulu. Jurnal Gradien, I(I), 4350. Retrieved from https://ejournal.unib.ac.id/index.php/gradien/article/view/I92

Adyana, M. (2012). Cara Pengolahan Obat Tradisional Baik dan Benar. Retrieved from http://www.herbaltarupratama.com/artikel-I8

Anisah, L.N. (202I). Hutan Sebagai Sumber Obat Masa Depan Perhutanan Sosial. Retrieved from http://kph.menlhk.go.id/sinpasdok/pages/lihat_berita/I59

Artha, P.T.Y.G. (2016). Studi Etnobotani Kawasan Masyarakat Lokal Desa Trunyan Provinsi Bali sebagai Buku Ajar pada Matakuliah Etnobotani di Perguruan Tinggi. Malang: Universitas Negeri Malang.

Depdikbud. (199I). Pengobatan Tradisional Pada Masyarakat Betawi Di Kelurahan Ciganjur. Dapertemen Pendidikan dan Kebudyaan. Retrieved from http://repositori.kemdikbud.go.id/8I84/I/Pengobatan\%20tradisional\%20pada\%20mas yarakat\%20betawi\%20di\%20kelurahan\%20ciganjur.Pdf

Dharmono. (2007). Kajian Etnobotani Tumbuhan Jalukap (Centella asiatika L.) di Suku Dayak Bukit Desa Haratai I Loksado. Bioscientiae, 4(2), 7I-78. Retrieved from https://ppip.ulm.ac.id/journals/index.php/bioscientiae/article/view/I65/I48

Efremila., Wardenaar, E., dan Sisilia, L. (2015). Studi Etnobotani Tumbuhan Obat oleh Suku Etnis Dayak di Desa Kayu Tanam Kecamatan Mandor Kabupaten Landak Kalimantan. Jurnal Hutan Lestari, 3(2) 234-246. Retrieved from http://dx.doi.org/I0.264I8/jhl.v3i2.I03I0

Hakim, M.A. (2014). Memperkuat Ketahanan Pangandemi Masa Depan Indonesia 2015-2025. Jakarta: Rumah Buku.

Hamidah, N., Rijanta, R., dan Marfai, M. A. (20I4). Kajian Transportasi Sungai Untuk Menghidupkan Kawasan Tepian Sungai Kahayan Kota Palangkaraya. Tata Loka, I6(I), II7. Retrieved from https://doi.org/IO.I47I0/tataloka.I6.I.I-I7

Harmida., Sarno., dan Yuni, V.F. (20II). Studi Etnofitomedika di Desa Lawang Agung Kecamatan Mulak Ulu Kabupaten Lahat Sumatera Selatan. Jurnal Penelitian Sains, I4(I), 42-46.Retrieved from https://doi.org/I0.26554/jps.vI4iI.I26

Haryono, D., Wardenaar, E., dan Yusro, F. (2014). Kajian Etnobotani Tumbuhan Obat di Desa Mengkiang Kecamatan Sanggau Kapuas Kabupaten Sanggau. Pontianak: Universitas Tanjungpura.

Herbie, T. (20I5). Kitab Tanaman Berkhasiat Obat-226 Tumbuhan Obat untuk Penyembuhan Penyakit dan Kebugaran Tubuh. Yogyakarta: Octopus Publihing House. 
Indra, Husni, H., dan Sisillia, L. (20I4). Kajian Etnobotani Tumbuhan Obat Etnis Melayu di Desa Sungai Baru dan Desa Sempadian Kabupaten Sambas. Jurnal Hutan Lestari, 2(2), I8I-I88. Retrieved from http://dx.doi.org/I0.264I8/jhl.v2i2.5839.

Julung, H., Supiandi,M.I., Ege, B., Mahanal, S., Zubaidah, S. (2018). Analisis Sumber Pengetahuan Tradisional Tanaman Obat yang Digunakan Oleh Masyarakat Suku Dayak Desa. Proceeding of Biology Education, 2(I), 67-74. Retrieved from https:// scholar.google.co.id/ citations?user=7otZpFEAAAAJ\&hl

Kandowangko, N.Y. (201I). Kajian Etnobotani Tanaman Obat oleh Masyarakat Kabupaten Bonebolango. Laporan Penelitian. Gorontalo: Universitas Negeri Gorontalo. Retrieved from https://repository.ung.ac.id/riset/show/2/737/kajian-etnobotani-tanaman-obatoleh masyarakatkabupaten-bonebolango-provinsi- gorontalo.html

Lestaridewi, N. K., Jamhari, M., Isnainar. (2017). Kajian Pemanfaatan Tanaman Sebagai Obat Tradisional di Desa Tolai Kecamatan Torue Kabupaten Parigi Moutong. E-JIP BIOL, 5(2), 92-I08. Retrieved from http://jurnal.untad.ac.id/jurnal/index.php/EBiol/issue/view/I3I4.

Moleong, L. J. (2010). Metodologi Penelitian Kualitatif. Bandung: Remaja Rosdakarya.

Munawaroh, V.F. (2012). Etnobotani Tumbuhan yang Bermanfaat untuk Kesehatan Reproduksi di Lingkungan Masyarakat Samin Kecamatan Margomulyo Kabupaten Bojonegoro. Malang: Universitas Islam Negeri Maulana Malik Ibrahim.

Nazir, M. (20I I). Metode Penelitian. Cetakan 6. Bogor: Ghalia Indonesia.

Noorcahyani, S. (2012). Tumbuhan Berkhasiat Obat Etnis Asli Kalimantan. Balikpapan Kalimantan Timur. Balikpapan: Balai Penelitian Teknologi Konservasi Sumber Daya Alam.

Nurhaida., Usman, F.H., dan Tavita, G.E. (2015). Studi Etnobotani Tumbuhan Obat di Dusun Kelampuk kecamatan Tanah Pinoh Barat Kabupaten Melawi. Jurnal Hutan Lestari, 3(4), 526-537. Retrieved from http://dx.doi.org/I0.264I8/jhl.v3i4.I26I2

Oktavia., Darma, I.D.P., dan Sujarwo, W. (2017). Studi Etnobotani Tumbuhan Obat di Kawasan Sekitar Danau Buyan-Tamblingan, Bali. Jurnal .krbogor.lipi.go.id, 20(I), I-I8. Retrieved from I0.I4203/bkr.v0i0.235.

Pardede, E. (2013). “Tinjauan Komposisi Kimia Buah dan Sayur: Peranan Sebagai Nutrisi dan Kaitannya dengan Teknologi Pengawetan dan Pengolahan”. Journal Visi, 2I(3), I-I6. Retrieved from kademik.uhn.ac.id/portal/public_html/JURNAL/JURNAL\%20ERIKA\%2OPARDED E/Tinjauan\%20Komposisi\%20Kimia\%20Buah\%20dan\%20Sayur\%20...\%20.pdf.

Rahayu, M., Sunarti, S., Sulistiarni, D., Prawiroatmodjo. (2006). Pengetahuan Tradisional dan Pemanfaatan Tumbuhan Obat Secara Tradisional Oleh Masyarakat Local di Pulau Wawonii, Sulawesi Tenggara. Jurnal Biodiversitas, 7(3), 245-250. Retrieved from https://media.neliti.com/media/publications/68590-ID-none.pdf

Riwut, T. ( I993). Kalimantan Membangun Alam dan Kebudayaan. Bioscientiae, 4(2), 7I78. Retrieved from https://ppip.ulm.ac.id/journals/index.php/bioscientiae/article/view/I65,

Silalahi, M. (2020). Diklat Etnobotani: Prodi Pendidikan Biologi Fakultas Keguruan dan Ilmu Pendidikan Universitas Kristen Indonesia. Retrieved from http://repository.uki.ac.id/I63I/I/Diktat\%20Etnobotani\%2025\% 20April\%202020.pdf.

Simamora, E.W. (2018). Potensi Tumbuhan Obat di Kawasan Hutan Lindung Harangan Bolak Kabupaten Samosir Provinsi Samosir. Skripsi, Universitas Sumatera Utara. 
Sofiah, W. (20I4). Studi Etnobotani Tumbuhan Obat pada Masyarakat Kecamatan Arjasa Kepulauan Kangean Kabupaten Sumenep Madura. Skripsi, Universitas Islam Negeri Maulana Malik Ibrahim Malang.

Supiandi, M.I., Julung, H., Ege, B., Mahanal,S., Zubaidah,S. (2020). Potential of Plant Roots as Traditional Medicines of the Dayak Desa Tribe in Pakak, West Kalimantan, Indonesia. Medicinal Plants, I2(2), 275-282. Retrieved from I0.5958/0975-6892.2020.00036.2

Supiandi,M.I \& Leliavia. (2020). The Source Traditional Medication Knowledge: The Dayak Iban in Lanjak Deras Village. JPBIO (Jurnal Pendidikan Biologi), 5(2), I84-I92. Retrieved from https://doi.org/I0.3I932/jpbio.v5i2.75I

Supiandi,M.I., Zubaidah, S., Mahanal, S., Julung, H., Ege, B. (2019). An Ethnobatical Study of Medicinal Plants Used by Dayak Desa from Tintau Menuah Forest and Genik Luak Bukit Bang. Medicinal Plants, II(3), 292-306. Retrieved from I0.5958/09756892.2019.00038.8

Supriadi, A. (2020). Rimpang Berkhasiat Jaga Daya Tahan Tubuh. Berita Kampus. Retrieved from https://pontianakpost.co.id/rimpang-jaga-daya-tahan-tubuh/

Suryadarma. (2008). Diklat Etnobotani. Yogyakarta: Jurusan Pendidikan Biologi FMIPA, Universitas Negeri Yogyakarta.

Syah, J., Usman, F.H., dan Yusro, F. (2014). Studi Etnobotani Tumbuhan Obat yang di Manfaatkan Masyarakat Dusun Nekbare Desa Babane Kecamatan Samalantan Kabupaten Bengkayang. Jurnal Hutan Lestari, 2(3), 419-426. Retrieved from http://dx.doi.org/I0.264I8/jhl.v2i3.7495

Uzlifah, U. (20I4). Aktivitas Antioksi dan Sirup Kombinasi Daun Sirsak (Anona muricata) dan Kulit Buah Naga (Hylocereus costaricensis) dengan Variasi Lama Perebusan. Skripsi. Surakarta: Universitas Muhammadiyah Surakarta.

Walujo, E.B. (20II). Sumbangan Ilmu Etnobotani dalam Memfasilitasi Hubungan Manusia dengan Tumbuhan dan Lingkungannya. Jurnal Biologi Indonesia, 7(2), 375-391. Retrieved from https://scholar.google.co.id/scholar?q=Sumbangan+Ilmu+Etnobotani+dalam+Memfasil itasi+Hubungan\&hl=en\&as_sdt $=0 \&$ as_vis $=I \&$ oi $=$ scholart

Wulandari, Fitmawati, dan Sofiyanti, N. (20I4). Eksplorasi Pengetahuan Tumbuhan Obat Etnis Sakai di Desa Petani, Duri-Riau. Jurnal Online Mahasiswa FMIPA, I(2), I-9. Retrieved from https://media.neliti.com/media/publications/I89706-ID-eksplorasi-pengetahuantumbuhan-obat-etn.pdf. 
Appendix I. List of medicinal plants in the Dayak Limbai tribe

\begin{tabular}{|c|c|c|c|c|c|c|}
\hline $\begin{array}{l}\mathrm{N} \\
\mathrm{o}\end{array}$ & Local Name & $\begin{array}{l}\text { Scientific } \\
\text { Name }\end{array}$ & Family & $\begin{array}{c}\text { Name of } \\
\text { disease }\end{array}$ & Parts Used & How to Use \\
\hline $\mathrm{I}$ & Kunyet & $\begin{array}{l}\text { Curcuma } \\
\text { domestica } \\
\text { Vahl }\end{array}$ & Zingiberaceae & Allergy & Rhizome & $\begin{array}{l}\text { Pounded with } \\
\text { rice, smeared on } \\
\text { the allergic part }\end{array}$ \\
\hline \multirow[t]{2}{*}{2} & Pakuk nait & $\begin{array}{l}\text { Stenochlaena } \\
\text { palustris } \\
\text { (Burm.) } \\
\text { Bedd }\end{array}$ & Blechnaceae & \multirow[t]{2}{*}{$\begin{array}{l}\text { Anemia } \\
\text { (lack of } \\
\text { blood) }\end{array}$} & \multirow[t]{2}{*}{ Leaf } & Vegetabled, eaten \\
\hline & Pelok & $\begin{array}{l}\text { Manihot } \\
\text { esculenta } \\
\text { Crantz }\end{array}$ & Euphorbiaceae & & & $\begin{array}{l}\text { Vegetabled, } \\
\text { boiled, eaten }\end{array}$ \\
\hline \multirow[t]{2}{*}{3} & Empuing & $\begin{array}{l}\text { Zingiber } \\
\text { purpureum } \\
\text { Roxb. }\end{array}$ & Zingiberaceae & \multirow[t]{2}{*}{ Gout } & \multirow[t]{2}{*}{ Rhizome } & $\begin{array}{l}\text { Pounded with } \\
\text { rice, smeared on } \\
\text { the gout part }\end{array}$ \\
\hline & Liok miroh & $\begin{array}{l}\text { Zingiber } \\
\text { officinale } \\
\text { Roxb. }\end{array}$ & & & & $\begin{array}{l}\text { Pounded, boiled, } \\
\text { drink }\end{array}$ \\
\hline 4 & $\begin{array}{l}\text { Pakuk } \\
\text { enturuk } \\
\text { halus }\end{array}$ & $\begin{array}{l}\text { Lygodium } \\
\text { flexuosum } \\
\text { (L.) Sw. }\end{array}$ & Schizaeaceae & $\begin{array}{l}\text { Acute } \\
\text { asthma }\end{array}$ & Leaf, root & Boiled, drink \\
\hline \multirow[t]{4}{*}{5} & Idu & $\begin{array}{l}\text { Centalla } \\
\text { asiatica (L.) } \\
\text { Urb. }\end{array}$ & Umbilliferae & \multirow[t]{4}{*}{$\begin{array}{l}\text { Swelling due } \\
\text { to insect } \\
\text { stings }\end{array}$} & Root & $\begin{array}{l}\text { Pounded, } \\
\text { smeared on the } \\
\text { part affected by } \\
\text { the sting }\end{array}$ \\
\hline & $\begin{array}{l}\text { Kayu patoh } \\
\text { tulong }\end{array}$ & $\begin{array}{l}\text { Euphorbia } \\
\text { tirucalli L. }\end{array}$ & Euphorbiaceae & & \multirow[t]{2}{*}{ Latex } & \multirow{2}{*}{$\begin{array}{l}\text { Smeared on the } \\
\text { part affected by } \\
\text { the sting }\end{array}$} \\
\hline & Keladi & $\begin{array}{l}\text { Colocasia } \\
\text { esculenta } \\
\text { (L.) Schott }\end{array}$ & Araceae & & & \\
\hline & Rangki alus & $\begin{array}{l}\text { Capsicum } \\
\text { frutescens } \mathrm{L} .\end{array}$ & Solanaceae & & Fruit & $\begin{array}{l}\text { Cutted, smeared } \\
\text { on the part } \\
\text { affected by the } \\
\text { sting }\end{array}$ \\
\hline \multirow[t]{2}{*}{6} & $\begin{array}{l}\text { Baong } \\
\text { lembak }\end{array}$ & $\begin{array}{l}\text { Eleutherine } \\
\text { americana } \\
\text { Merr. }\end{array}$ & Iridaceae & \multirow[t]{2}{*}{ Dysentery } & $\begin{array}{l}\text { Bulbs } \\
\text { (tubers) }\end{array}$ & Boiled, drink \\
\hline & Gandis & $\begin{array}{l}\text { Garcinia } \\
\text { xanthochym } \\
\text { us Hook. f. } \\
\text { ex T. } \\
\text { Anderson }\end{array}$ & Clusiaceae & & Bark & $\begin{array}{l}\text { Scraped, boiled, } \\
\text { drink }\end{array}$ \\
\hline \multirow[t]{2}{*}{7} & Baong putih & $\begin{array}{l}\text { Allium } \\
\text { sativum L. }\end{array}$ & Alliaceae & Blain & Tuber & $\begin{array}{l}\text { Smeared around } \\
\text { blain }\end{array}$ \\
\hline & Pisong emas & $\begin{array}{l}\text { Musa } \\
\text { acuminata L. }\end{array}$ & Musaceae & & Shoots & $\begin{array}{l}\text { Pounded, } \\
\text { smeared on blain }\end{array}$ \\
\hline
\end{tabular}




\begin{tabular}{|c|c|c|c|c|c|c|}
\hline 8 & Cekur & $\begin{array}{l}\text { Kaempferia } \\
\text { galanga L. }\end{array}$ & Zingiberaceae & Wormy & $\begin{array}{l}\text { Rhizome, } \\
\text { leaf }\end{array}$ & Eaten right away \\
\hline 9 & Paoh & $\begin{array}{l}\text { Mangifera } \\
\text { timorensis } \\
\text { Blume }\end{array}$ & Anacardiaceae & Hiccup & Bark & $\begin{array}{l}\text { Scraped, boiled, } \\
\text { drink }\end{array}$ \\
\hline \multirow[t]{3}{*}{ IO } & Kabu & $\begin{array}{l}\text { Ceiba } \\
\text { pentandra } \\
\text { (L.) Gaertn. }\end{array}$ & Malvaceae & \multirow[t]{3}{*}{ Fever } & \multirow[t]{3}{*}{ Leaf } & $\begin{array}{l}\text { Pounded with } \\
\text { rice, smeared all } \\
\text { over the body }\end{array}$ \\
\hline & $\begin{array}{l}\text { Komong } \\
\text { sepatu }\end{array}$ & $\begin{array}{l}\text { Hibiscus } \\
\text { rosa sinensis } \\
\text { L. }\end{array}$ & & & & \\
\hline & $\begin{array}{l}\text { Rumput } \\
\text { ngamenano } \\
\text { k }\end{array}$ & $\begin{array}{l}\text { Phyllanthus } \\
\text { urinaria L. }\end{array}$ & Phyllanthaceae & & & \\
\hline \multirow[t]{3}{*}{ II } & Jambu biji & $\begin{array}{l}\text { Psidium } \\
\text { guajava L. }\end{array}$ & Myrtaceae & \multirow[t]{3}{*}{ Diarrhea } & Leaf & Boiled, drink \\
\hline & Lajok & $\begin{array}{l}\text { Alpinia } \\
\text { galanga (L.) } \\
\text { Willd. }\end{array}$ & Zingiberaceae & & Rhizome & $\begin{array}{l}\text { Pounded, boiled, } \\
\text { drink }\end{array}$ \\
\hline & Liok miroh & $\begin{array}{l}\text { Zingiber } \\
\text { officinale } \\
\text { Roxb. }\end{array}$ & & & & \\
\hline $\mathrm{I} 2$ & Empuing & $\begin{array}{l}\text { Zingiber } \\
\text { purpureum } \\
\text { Roxb. }\end{array}$ & Zingiberaceae & $\begin{array}{l}\text { Dysentery } \\
\text { (intestinal } \\
\text { infection) } \\
\end{array}$ & Bud eye & $\begin{array}{l}\text { Burned, taped to } \\
\text { the anus }\end{array}$ \\
\hline \multirow[t]{2}{*}{ I3 } & $\begin{array}{l}\text { Entimon } \\
\text { batong }\end{array}$ & $\begin{array}{l}\text { Cucumis } \\
\text { sativus L. }\end{array}$ & Cucurbitaceae & \multirow[t]{2}{*}{$\begin{array}{l}\text { Black spots } \\
\text { on face }\end{array}$} & Fruit & $\begin{array}{l}\text { Cutted, smeared } \\
\text { on the face }\end{array}$ \\
\hline & $\begin{array}{l}\text { Pakuk } \\
\text { pantai }\end{array}$ & $\begin{array}{l}\text { Diplazium } \\
\text { esculentum } \\
\text { Schwartz. } \\
\end{array}$ & Athyriaceae & & Stem, leaf & $\begin{array}{l}\text { Pounded, } \\
\text { smeared on the } \\
\text { face }\end{array}$ \\
\hline \multirow[t]{2}{*}{$\mathrm{I} 4$} & Kemaounk & $\begin{array}{l}\text { Piper betle } \\
\text { L. }\end{array}$ & Piperaceae & \multirow[t]{2}{*}{ Itchy redness } & \multirow[t]{2}{*}{ Leaf } & $\begin{array}{l}\text { Kneaded, } \\
\text { smeared on the } \\
\text { itchy part when } \\
\text { bathing }\end{array}$ \\
\hline & Puring & $\begin{array}{l}\text { Codiaemum } \\
\text { variegatum } \\
\text { A. Juss. }\end{array}$ & Euphorbiaceae & & & $\begin{array}{l}\text { Pounded with } \\
\text { rice, smeared on } \\
\text { the itchy part }\end{array}$ \\
\hline 15 & Kobajaroh & $\begin{array}{l}\text { Hyptis } \\
\text { capitata Jacq. }\end{array}$ & Lamiaceae & $\begin{array}{l}\text { Swollen } \\
\text { gums }\end{array}$ & Leaf & $\begin{array}{l}\text { Pounded with } \\
\text { rice, smeared on } \\
\text { the swollen gums }\end{array}$ \\
\hline 16 & Kunyet & $\begin{array}{l}\text { Curcuma } \\
\text { domestica } \\
\text { Vahl. } \\
\end{array}$ & Zingiberaceae & $\begin{array}{l}\text { Nasal } \\
\text { congetion }\end{array}$ & Rhizome & $\begin{array}{l}\text { Smeared on the } \\
\text { bridge of the } \\
\text { nose }\end{array}$ \\
\hline I7 & Empuing & $\begin{array}{l}\text { Zingiber } \\
\text { purpureum } \\
\text { Roxb. }\end{array}$ & Zingiberaceae & $\begin{array}{l}\text { Hernia } \\
\text { (dropped } \\
\text { down) }\end{array}$ & $\begin{array}{l}\text { Rhizome, } \\
\text { shoots }\end{array}$ & $\begin{array}{l}\text { Burned, smeared } \\
\text { on the lump }\end{array}$ \\
\hline 18 & Baong putih & $\begin{array}{l}\text { Allium } \\
\text { sativum } \mathrm{L} .\end{array}$ & Alliaceae & $\begin{array}{l}\text { Hypertensio } \\
\mathrm{n} \text { (High }\end{array}$ & Tuber & $\begin{array}{l}\text { Boiled, drink ( } 5 \\
\text { cups to } 2 \text { cups) }\end{array}$ \\
\hline
\end{tabular}




\begin{tabular}{|c|c|c|c|c|c|c|}
\hline & & & & \multirow{4}{*}{$\begin{array}{l}\text { Blood } \\
\text { Pressure) }\end{array}$} & \multirow{4}{*}{ Leaf } & \multirow{4}{*}{ Boiled, drink } \\
\hline & $\begin{array}{l}\text { Belimbing } \\
\text { tunjuk }\end{array}$ & $\begin{array}{l}\text { Averrhoea } \\
\text { bilimbi L. }\end{array}$ & Oxalidaceae & & & \\
\hline & Mengkudu & $\begin{array}{l}\text { Morinda } \\
\text { citrifolia L. }\end{array}$ & Rubiaceae & & & \\
\hline & Pepaya & $\begin{array}{l}\text { Carica } \\
\text { papaya L. }\end{array}$ & Caricaceae & & & \\
\hline \multirow[t]{2}{*}{19} & $\begin{array}{l}\text { Pelok } \\
\text { rampuk }\end{array}$ & $\begin{array}{l}\text { Ipomoea } \\
\text { batatas Lam. }\end{array}$ & Convolvulaceae & \multirow[t]{2}{*}{ Breast cancer } & \multirow[t]{2}{*}{ Leaf } & \multirow{2}{*}{$\begin{array}{l}\text { Pounded with } \\
\text { rice, smeared on } \\
\text { the breast }\end{array}$} \\
\hline & $\begin{array}{l}\text { Puring } \\
\text { miroh }\end{array}$ & $\begin{array}{l}\text { Codiaemum } \\
\text { variegatum } \\
\text { A. Juss. }\end{array}$ & Euphorbiaceae & & & \\
\hline \multirow[t]{2}{*}{20} & Nyiur & $\begin{array}{l}\text { Cocos } \\
\text { nucifera L. }\end{array}$ & Arecaceae & \multirow[t]{2}{*}{$\begin{array}{l}\text { Food } \\
\text { poisoning }\end{array}$} & $\begin{array}{l}\text { Coconut } \\
\text { water }\end{array}$ & Drink right away \\
\hline & Pasok bumi & $\begin{array}{l}\text { Eurycoma } \\
\text { longifolia } \\
\text { Jack } \\
\end{array}$ & Simaroubaceae & & Root & Boiled, drink \\
\hline \multirow[t]{2}{*}{$2 \mathrm{I}$} & Empuing & $\begin{array}{l}\text { Zingiber } \\
\text { purpureum } \\
\text { Roxb. }\end{array}$ & Zingiberaceae & \multirow[t]{2}{*}{ Sprain } & \multirow[t]{2}{*}{ Rhizome } & $\begin{array}{l}\text { Pounded with } \\
\text { rice, smeared on } \\
\text { the sprained part }\end{array}$ \\
\hline & Kunyet & $\begin{array}{l}\text { Curcuma } \\
\text { domestica } \\
\text { Vahl. }\end{array}$ & & & & $\begin{array}{l}\text { Burned, pounded } \\
\text { with rice, } \\
\text { smeared on the } \\
\text { sprained part }\end{array}$ \\
\hline 22 & Nangkok & $\begin{array}{l}\text { Artocarpus } \\
\text { heterophyllu } \\
s \text { Lam. }\end{array}$ & Moraceae & Water flea & Dry leaf & $\begin{array}{l}\text { Burned to ashes, } \\
\text { mixed with } \\
\text { cooking oil, } \\
\text { smeared on the } \\
\text { part affected by } \\
\text { water fleas }\end{array}$ \\
\hline 23 & Nangkok & $\begin{array}{l}\text { Artocarpus } \\
\text { heterophyllu } \\
s \text { Lam. }\end{array}$ & Moraceae & $\begin{array}{l}\text { Scratched by } \\
\text { falling }\end{array}$ & Dry leaf & $\begin{array}{l}\text { Burned to ashes, } \\
\text { mixed with } \\
\text { cooking oil, } \\
\text { smeared on the } \\
\text { blisters }\end{array}$ \\
\hline 24 & Jungka & $\begin{array}{l}\text { Crinum } \\
\text { Asiaticum } \\
\text { L. }\end{array}$ & Amaryllidaceae & Liver & Leaf & $\begin{array}{l}\text { Pounded, } \\
\text { smeared on the } \\
\text { chest to the } \\
\text { stomach }\end{array}$ \\
\hline & Kunyet & $\begin{array}{l}\text { Curcuma } \\
\text { domestica } \\
\text { Vahl. }\end{array}$ & Zingiberaceae & & Rhizome & $\begin{array}{l}\text { Pounded with } \\
\text { rice, smeared on } \\
\text { the chest to the } \\
\text { stomach }\end{array}$ \\
\hline & Pinong & $\begin{array}{l}\text { Areca } \\
\text { catechu L. }\end{array}$ & Arecaceae & & Leaf & $\begin{array}{l}\text { Heated in the } \\
\text { fireplace, taped } \\
\text { on the chest to } \\
\text { the stomach }\end{array}$ \\
\hline
\end{tabular}




\begin{tabular}{|c|c|c|c|c|c|c|}
\hline & $\begin{array}{l}\text { Terong } \\
\text { tulih }\end{array}$ & $\begin{array}{l}\text { Solanum } \\
\text { torvum } \mathrm{Sw} .\end{array}$ & Solanaceae & & & $\begin{array}{l}\text { Pounded, mixed } \\
\text { with slices of } \\
\text { wooden cauldron } \\
\text { lid, sliced wood } \\
\text { chili pounder and } \\
\text { added soot from } \\
\text { the top of the } \\
\text { wood stove, plus } \\
\text { steamed rice } \\
\text { (burned), } \\
\text { smeared on the } \\
\text { chest to the } \\
\text { stomach }\end{array}$ \\
\hline 25 & $\begin{array}{l}\text { Engkereban } \\
\mathrm{g}\end{array}$ & $\begin{array}{l}\text { Psyhotria } \\
\text { vividifloria } \\
\text { Reinw. ex } \\
\text { Blume }\end{array}$ & Rubiaceae & \multirow[t]{4}{*}{ Burns } & Leaf & $\begin{array}{l}\text { Pounded, taped } \\
\text { to the burnt area }\end{array}$ \\
\hline & Keladi & $\begin{array}{l}\text { Colocasia } \\
\text { esculenta var. } \\
\text { esculanta } \\
\text { (L.) Schott }\end{array}$ & Araceae & & Latex & $\begin{array}{l}\text { Taped to the } \\
\text { burnt area }\end{array}$ \\
\hline & Ngingo & $\begin{array}{l}\text { Psychotria } \\
\text { nervosa Sw. }\end{array}$ & Rubiaceae & & Leaf & $\begin{array}{l}\text { Pounded, taped } \\
\text { to the burnt area }\end{array}$ \\
\hline & $\begin{array}{l}\text { Rumput } \\
\text { sapi }\end{array}$ & $\begin{array}{l}\text { Paspalum } \\
\text { conjugatum } \\
\text { Berggr. }\end{array}$ & $\begin{array}{l}\text { Poaceae } \\
\text { (Gramineae) }\end{array}$ & & & \\
\hline 26 & Selasih & $\begin{array}{l}\text { Ocimum } \\
\text { basilicum L. }\end{array}$ & Lamiaceae & Deep wound & Leaf, fruit & Boiled, drink \\
\hline \multirow[t]{3}{*}{27} & Empuing & $\begin{array}{l}\text { Zingiber } \\
\text { purpureum } \\
\text { Roxb. }\end{array}$ & Zingiberaceae & \multirow[t]{3}{*}{ Paralysed } & Rhizome & \multirow{3}{*}{$\begin{array}{l}\text { Pounded with } \\
\text { rice, smeared on } \\
\text { the paralyzed } \\
\text { body part }\end{array}$} \\
\hline & Kepuak & $\begin{array}{l}\text { Artocarpus } \\
\text { elasticus } \\
\text { Reinw. ex } \\
\text { Blume }\end{array}$ & Moraceae & & Young leaf & \\
\hline & Kunyet & $\begin{array}{l}\text { Curcuma } \\
\text { domestica } \\
\text { Vahl. } \\
\end{array}$ & Zingiberaceae & & Rhizome & \\
\hline \multirow[t]{2}{*}{28} & Gelinggam & $\begin{array}{l}\text { Cassia alata } \\
\text { L. }\end{array}$ & Leguminosae & \multirow[t]{2}{*}{ Ulcer } & Leaf & Pounded, drink \\
\hline & $\begin{array}{l}\text { Terong } \\
\text { tulih }\end{array}$ & $\begin{array}{l}\text { Solanum } \\
\text { torvum Sw. }\end{array}$ & Solanaceae & & Fruit & Raw eaten \\
\hline \multirow[t]{2}{*}{29} & Kelopuk & $\begin{array}{l}\text { Nauclea } \\
\text { speciosa }\end{array}$ & Rubiaceae & \multirow[t]{2}{*}{ Malaria } & Fruit & Raw eaten \\
\hline & $\begin{array}{l}\text { Pasok } \\
\text { matohari }\end{array}$ & $\begin{array}{l}\text { Clidemia } \\
\text { hirta (L.) } \\
\text { D.Don }\end{array}$ & $\begin{array}{l}\text { Melastomatacea } \\
e\end{array}$ & & Root, bark & Boiled, drink \\
\hline
\end{tabular}




\begin{tabular}{|c|c|c|c|c|c|c|}
\hline & Pepaya & $\begin{array}{l}\text { Carica } \\
\text { papaya L. }\end{array}$ & Caricaceae & & Leaf & \\
\hline \multirow[t]{2}{*}{30} & Arum & $\begin{array}{l}\text { Amaranthus } \\
\text { spinosus L. }\end{array}$ & Amaranthaceae & $\begin{array}{l}\text { Malnutrition } \\
\text { (lack of } \\
\text { nutrition) }\end{array}$ & Leaf, stem & Vegetabled, eaten \\
\hline & Pelok & $\begin{array}{l}\text { Manihot } \\
\text { esculenta } \\
\text { Crantz }\end{array}$ & Euphorbiaceae & & Leaf & $\begin{array}{l}\text { Pounded, } \\
\text { vegetabled, eaten }\end{array}$ \\
\hline $3 \mathrm{I}$ & Empuing & $\begin{array}{l}\text { Zingiber } \\
\text { purpureum } \\
\text { Roxb. }\end{array}$ & Zingiberaceae & $\begin{array}{l}\text { Bruised from } \\
\text { falling }\end{array}$ & Rhizome & $\begin{array}{l}\text { Pounded with } \\
\text { rice, smeared on } \\
\text { the bruise }\end{array}$ \\
\hline \multirow[t]{5}{*}{32} & Kelopuk & $\begin{array}{l}\text { Nauclea } \\
\text { speciosa }\end{array}$ & Rubiaceae & $\begin{array}{l}\text { Accelerate } \\
\text { breast } \\
\text { feeding }\end{array}$ & Leaf & Vegetabled, eaten \\
\hline & Konong & $\begin{array}{l}\text { Ficus } \\
\text { variegata } \\
\text { Blume } \\
\end{array}$ & Moraceae & & $\begin{array}{l}\text { Leaf, } \\
\text { young stem }\end{array}$ & \\
\hline & Nyiur & $\begin{array}{l}\text { Cocos } \\
\text { nucifera L. }\end{array}$ & Arecaceae & & $\begin{array}{l}\text { Young } \\
\text { flower, } \\
\text { shoots }\end{array}$ & \\
\hline & $\begin{array}{l}\text { Pakuk } \\
\text { korok }\end{array}$ & $\begin{array}{l}\text { Polypodium } \\
\text { verrucosum } \\
\text { Hook. }\end{array}$ & $\begin{array}{l}\text { Nephrolepidace } \\
\text { ae }\end{array}$ & & $\begin{array}{l}\text { Young } \\
\text { stem, leaf }\end{array}$ & \\
\hline & $\begin{array}{l}\text { Pelok } \\
\text { rampuk }\end{array}$ & $\begin{array}{l}\text { Ipomoea } \\
\text { batatas Lam. }\end{array}$ & Convolvulaceae & & Latex & $\begin{array}{l}\text { Smeared on the } \\
\text { breast }\end{array}$ \\
\hline \multirow[t]{3}{*}{33} & Kedaung & $\begin{array}{l}\text { Parkia } \\
\text { javanica } \\
\text { (Lam.) } \\
\text { Merr. } \\
\end{array}$ & Fabaceae & $\begin{array}{l}\text { Accelerate } \\
\text { defecate }\end{array}$ & Ripe fruit & Eaten right away \\
\hline & Pepaya & $\begin{array}{l}\text { Carica } \\
\text { papaya L. }\end{array}$ & Caricaceae & & & \\
\hline & Pisang & $\begin{array}{l}\text { Musa } \\
\text { paradisiaca } \\
\text { L. }\end{array}$ & Musaceae & & & \\
\hline 34 & Putri malu & $\begin{array}{l}\text { Mimosa } \\
\text { pudica L. }\end{array}$ & Fabaceae & $\begin{array}{l}\text { Accelerate } \\
\text { and reduce } \\
\text { menstrual } \\
\text { pain }\end{array}$ & Leaf & $\begin{array}{l}\text { Pounded with } \\
\text { rice, smeared } \\
\text { around the vagina }\end{array}$ \\
\hline 35 & Lajok & $\begin{array}{l}\text { Alpinia } \\
\text { galanga (L.) } \\
\text { Willd }\end{array}$ & Zingiberaceae & $\begin{array}{l}\text { Make birth } \\
\text { easier }\end{array}$ & Rhizome & $\begin{array}{l}\text { Pounded with } \\
\text { rice, smeared on } \\
\text { the stomach and } \\
\text { around the vagina }\end{array}$ \\
\hline 36 & Empuing & $\begin{array}{l}\text { Zingiber } \\
\text { purpureum } \\
\text { Roxb. }\end{array}$ & Zingiberaceae & $\begin{array}{l}\text { Overcoming } \\
\text { prickly heat }\end{array}$ & Rhizome & $\begin{array}{l}\text { Pounded with } \\
\text { rice, smeared on } \\
\text { the part affected } \\
\text { by prickly heat }\end{array}$ \\
\hline 37 & Padi & Oryza sativa & Poaceae & Overcoming & Old fruit & Pounded (mixed \\
\hline
\end{tabular}




\begin{tabular}{|c|c|c|c|c|c|c|}
\hline & & L. & (Gramineae) & fatigue & & $\begin{array}{l}\text { empuing rhizome } \\
\text { and red ginger), } \\
\text { drink }\end{array}$ \\
\hline \multirow[t]{2}{*}{38} & Ati-ati & $\begin{array}{l}\text { Plectranthus } \\
\text { scutellarioide } \\
s \text { (L.) R.Br }\end{array}$ & Lamiaceae & \multirow[t]{2}{*}{$\begin{array}{l}\text { Treat } \\
\text { miscarriage }\end{array}$} & \multirow[t]{2}{*}{ Stem, leaf } & \multirow[t]{2}{*}{$\begin{array}{l}\text { Pounded with } \\
\text { rice, smeared } \\
\text { around the vagina }\end{array}$} \\
\hline & $\begin{array}{l}\text { Pakuk } \\
\text { pantai }\end{array}$ & $\begin{array}{l}\text { Diplazium } \\
\text { esculentum } \\
\text { Schwartz. }\end{array}$ & Athyriaceae & & & \\
\hline 39 & Tembulan & $\begin{array}{l}\text { Lansium } \\
\text { domesticum } \\
\text { var. aquaeum }\end{array}$ & Meliaceae & $\begin{array}{l}\text { Fever (high } \\
\text { fever, chills) }\end{array}$ & Seed, rind & Eaten right away \\
\hline 40 & $\begin{array}{l}\text { Terong } \\
\text { tulih }\end{array}$ & $\begin{array}{l}\text { Solanum } \\
\text { torvum } \mathrm{Sw} .\end{array}$ & Solanaceae & Nauseous & Fruit & Raw eaten \\
\hline \multirow[t]{3}{*}{$4 \mathrm{I}$} & $\begin{array}{l}\text { Jerangau } \\
\text { miroh }\end{array}$ & Acorus sp & Acoraceae & \multirow[t]{3}{*}{$\begin{array}{l}\text { Vomiting } \\
\text { blood }\end{array}$} & Rhizome & $\begin{array}{l}\text { Pounded, drink } \\
\text { or raw eaten }\end{array}$ \\
\hline & Pasok bumi & $\begin{array}{l}\text { Eurycoma } \\
\text { longifolia } \\
\text { Jack }\end{array}$ & Simaroubaceae & & Root, bark & Boiled, drink \\
\hline & Tembulan & $\begin{array}{l}\text { Lansium } \\
\text { domesticum } \\
\text { var. aquaeum }\end{array}$ & Meliaceae & & Root, rind & \\
\hline \multirow[t]{3}{*}{42} & Benalu & Loranthus L & Loranthaceae & \multirow[t]{3}{*}{$\begin{array}{l}\text { Stomach } \\
\text { pain }\end{array}$} & \multirow[t]{3}{*}{ Leaf } & \multirow{3}{*}{$\begin{array}{l}\text { Pounded with } \\
\text { rice, smeared on } \\
\text { the stomach }\end{array}$} \\
\hline & Putri malu & $\begin{array}{l}\text { Mimosa } \\
\text { pudica L. }\end{array}$ & Fabaceae & & & \\
\hline & Sisit nago & $\begin{array}{l}\text { Drymoglosu } \\
m \\
\text { piloselloides } \\
\text { (L.) Presl. }\end{array}$ & Polypodiaceae & & & \\
\hline \multirow[t]{2}{*}{43} & Empuing & $\begin{array}{l}\text { Zingiber } \\
\text { purpureum } \\
\text { Roxb. }\end{array}$ & Zingiberaceae & \multirow[t]{2}{*}{ Back pain } & \multirow[t]{2}{*}{ Rhizome } & \multirow[t]{2}{*}{$\begin{array}{l}\text { Pounded with } \\
\text { rice, smeared on } \\
\text { the back }\end{array}$} \\
\hline & Kunyet & $\begin{array}{l}\text { Curcuma } \\
\text { domestica } \\
\text { Vahl. } \\
\end{array}$ & & & & \\
\hline 44 & Baong kucai & $\begin{array}{l}\text { Allium } \\
\text { schoenopras } \\
\text { um Rottler } \\
\text { ex Spreng. } \\
\text { dan A. } \\
\text { Ramous }\end{array}$ & Alliaceae & \multirow[t]{3}{*}{$\begin{array}{l}\text { Postpartum } \\
\text { (thin, black, } \\
\text { lethargic, } \\
\text { likes to } \\
\text { sleep) }\end{array}$} & $\begin{array}{l}\text { Bulbs } \\
\text { (tubers) }\end{array}$ & \multirow[t]{2}{*}{$\begin{array}{l}\text { Pounded with } \\
\text { rice, smeared } \\
\text { around the vagina }\end{array}$} \\
\hline & Kunyet & $\begin{array}{l}\text { Curcuma } \\
\text { domestica } \\
\text { Vahl. } \\
\end{array}$ & Zingiberaceae & & Rhizome & \\
\hline & Liok miroh & $\begin{array}{l}\text { Zingiber } \\
\text { officinale } \\
\text { Roxb. var. }\end{array}$ & & & & $\begin{array}{l}\text { Pounded, boiled, } \\
\text { drink }\end{array}$ \\
\hline
\end{tabular}




\begin{tabular}{|c|c|c|c|c|c|c|}
\hline & & $\begin{array}{l}\text { rubrum } \\
\text { Rosc }\end{array}$ & & & & \\
\hline & $\begin{array}{l}\text { Pakuk } \\
\text { rabun }\end{array}$ & $\begin{array}{l}\text { Lycopodium } \\
\text { cernuum L. }\end{array}$ & Lycopodiaceae & & Root & $\begin{array}{l}\text { Pounded with } \\
\text { rice, smeared } \\
\text { around the vagina }\end{array}$ \\
\hline 45 & Kayu aro & $\begin{array}{l}\text { Ficus } \\
\text { benjamina L }\end{array}$ & Moraceae & Fracture & Bark & $\begin{array}{l}\text { Woven, then } \\
\text { wrapped in a } \\
\text { broken place } \\
\text { (mixed with } \\
\text { chopped chicks, } \\
\text { honey, free-range } \\
\text { chicken eggs) }\end{array}$ \\
\hline \multirow[t]{2}{*}{46} & $\begin{array}{l}\text { Eceng } \\
\text { gondok }\end{array}$ & $\begin{array}{l}\text { Eichhornia } \\
\text { crassipes } \\
\text { (Mart.) } \\
\text { Solms. }\end{array}$ & Pontederiaceae & \multirow[t]{2}{*}{$\begin{array}{l}\text { Breast } \\
\text { enlargement }\end{array}$} & \multirow[t]{2}{*}{ Leaf } & \multirow[t]{2}{*}{$\begin{array}{l}\text { Pounded with } \\
\text { rice, smeared on } \\
\text { the breast }\end{array}$} \\
\hline & $\begin{array}{l}\text { Pelok } \\
\text { rampuk }\end{array}$ & $\begin{array}{l}\text { Ipomoea } \\
\text { batatas Lam. }\end{array}$ & Convolvulaceae & & & \\
\hline \multirow[t]{2}{*}{47} & Jungka & $\begin{array}{l}\text { Crinum } \\
\text { asiaticum L. }\end{array}$ & Amaryllidaceae & \multirow[t]{2}{*}{$\begin{array}{l}\text { Testicular } \\
\text { enlargement }\end{array}$} & Leaf & \multirow[t]{2}{*}{$\begin{array}{l}\text { Pounded with } \\
\text { rice, smeared on } \\
\text { the testicles }\end{array}$} \\
\hline & Kunyet & $\begin{array}{l}\text { Curcuma } \\
\text { domestica } \\
\text { Vahl. }\end{array}$ & Zingiberaceae & & Rhizome & \\
\hline \multirow[t]{5}{*}{48} & Ati-ati & $\begin{array}{l}\text { Plectranthus } \\
\text { scutellarioide } \\
s \text { (L.) R.Br. }\end{array}$ & Lamiaceae & \multirow[t]{5}{*}{ Bleeding } & $\begin{array}{l}\text { Leaf, } \\
\text { young stem }\end{array}$ & \multirow[t]{5}{*}{$\begin{array}{l}\text { Pounded with } \\
\text { rice, smeared } \\
\text { around the vagina }\end{array}$} \\
\hline & $\begin{array}{l}\text { Engkereban } \\
\mathrm{g}\end{array}$ & $\begin{array}{l}\text { Psyhotria } \\
\text { vividifloria } \\
\text { Reinw. ex } \\
\text { Blume } \\
\end{array}$ & Rubiaceae & & Leaf & \\
\hline & $\begin{array}{l}\text { Pakuk } \\
\text { korok }\end{array}$ & $\begin{array}{l}\text { Polypodium } \\
\text { verrucosum } \\
\text { Hook. }\end{array}$ & $\begin{array}{l}\text { Nephrolepidace } \\
\text { ae }\end{array}$ & & & \\
\hline & $\begin{array}{l}\text { Pakuk } \\
\text { pantai }\end{array}$ & $\begin{array}{l}\text { Diplazium } \\
\text { esculentum } \\
\text { Schwartz }\end{array}$ & Athyriaceae & & Stem, leaf & \\
\hline & $\begin{array}{l}\text { Tobu (the } \\
\text { one with } \\
\text { bear bite } \\
\text { marks) }\end{array}$ & $\begin{array}{l}\text { Saccharum } \\
\text { officinarum } \\
\text { L. }\end{array}$ & $\begin{array}{l}\text { Poaceae } \\
\text { (Gramineae) }\end{array}$ & & Stem dregs & \\
\hline \multirow[t]{2}{*}{49} & Cahong & $\begin{array}{l}\text { Piper nigrum } \\
\mathrm{L}\end{array}$ & Piperaceae & \multirow[t]{2}{*}{ Impotent } & Dry fruit & $\begin{array}{l}\text { Pounded ( } 7 \\
\text { items). smeared } \\
\text { on the penis }\end{array}$ \\
\hline & Tebelian & $\begin{array}{l}\text { Eusideroxylo } \\
\text { n zwageri }\end{array}$ & Lauraceae & & $\begin{array}{l}\text { Leaf } \\
\text { (handful) }\end{array}$ & $\begin{array}{l}\text { Cut the tip and } \\
\text { base, take the }\end{array}$ \\
\hline
\end{tabular}




\begin{tabular}{|c|c|c|c|c|c|c|}
\hline & & $\begin{array}{l}\text { Teijsm. et } \\
\text { Binn. }\end{array}$ & & & & $\begin{array}{l}\text { middle, pounded } \\
\text { and smeared on } \\
\text { the penis }\end{array}$ \\
\hline \multirow[t]{5}{*}{50} & $\begin{array}{l}\text { Belimbing } \\
\text { tunjuk }\end{array}$ & $\begin{array}{l}\text { Averthoea } \\
\text { bilimbi L. }\end{array}$ & Oxalidaceae & \multirow{5}{*}{$\begin{array}{l}\text { Heart } \\
\text { disease and } \\
\text { cholesterol }\end{array}$} & Leaf & Boiled, drink \\
\hline & Cengkeh & $\begin{array}{l}\text { Syzygium } \\
\text { aromaticum } \\
\text { (L.) Merr. } \\
\text { dan L.M. } \\
\text { Perry }\end{array}$ & Myrtaceae & & Old fruit & $\begin{array}{l}\text { Boiled ( } 5 \text { cups to } \\
2 \text { cups), drink }\end{array}$ \\
\hline & Jolik & Zea mays L. & $\begin{array}{l}\text { Poaceae } \\
\text { (Gramineae) }\end{array}$ & & $\begin{array}{l}\text { Old corn } \\
\text { hair } \\
\text { (sufficientl } \\
\text { y) }\end{array}$ & Boiled, drink \\
\hline & Manggo & $\begin{array}{l}\text { Mangifera } \\
\text { indica L. }\end{array}$ & Anacardiaceae & & $\begin{array}{l}\text { Dry leaf } \\
\text { (handful) }\end{array}$ & \\
\hline & Pisong & $\begin{array}{l}\text { Musa } \\
\text { paradisiaca } \\
\text { L. }\end{array}$ & Musaceae & & & \\
\hline \multirow[t]{2}{*}{$5 \mathrm{I}$} & Gelinggam & $\begin{array}{l}\text { Cassia alata } \\
\text { L. }\end{array}$ & Leguminosae & \multirow{2}{*}{$\begin{array}{l}\text { Skin disease } \\
\text { (Tinea } \\
\text { versicolor) }\end{array}$} & Leaf & \multirow{2}{*}{$\begin{array}{l}\text { Pounded, } \\
\text { smeared on the } \\
\text { part that has } \\
\text { Tinea versicolor }\end{array}$} \\
\hline & Lajok & $\begin{array}{l}\text { Alpinia } \\
\text { galanga (L.) } \\
\text { Willd. }\end{array}$ & Zingiberaceae & & Rhizome & \\
\hline \multirow[t]{3}{*}{52} & $\begin{array}{l}\text { Akar } \\
\text { kuning }\end{array}$ & $\begin{array}{l}\text { Arcangelisisa } \\
\text { flava L. }\end{array}$ & $\begin{array}{l}\text { Menispermacea } \\
e\end{array}$ & \multirow[t]{3}{*}{ Hepatitis } & Root & \multirow[t]{2}{*}{ Boiled, drink } \\
\hline & Engkabong & $\begin{array}{l}\text { Shorea } \\
\text { beccariana } \\
\text { Roxb. ex } \\
\text { Gaertn. }\end{array}$ & $\begin{array}{l}\text { Dipterocarpacea } \\
e\end{array}$ & & Leaf & \\
\hline & Liok miroh & $\begin{array}{l}\text { Zingiber } \\
\text { officinale } \\
\text { Roxb. var. } \\
\text { rubrum } \\
\text { Rosc }\end{array}$ & Zingiberaceae & & Rhizome & $\begin{array}{l}\text { Pounded, boiled, } \\
\text { drink }\end{array}$ \\
\hline \multirow[t]{2}{*}{53} & Cekur & $\begin{array}{l}\text { Kaempferia } \\
\text { galanga L. }\end{array}$ & Zingiberaceae & \multirow[t]{2}{*}{$\begin{array}{l}\text { Flatulence in } \\
\text { children }\end{array}$} & \multirow[t]{2}{*}{ Rhizome } & \multirow[t]{2}{*}{$\begin{array}{l}\text { Chewed, sprayed } \\
\text { on the stomach }\end{array}$} \\
\hline & $\begin{array}{l}\text { Jerangau } \\
\text { putih }\end{array}$ & $\begin{array}{l}\text { Acorus } \\
\text { calamus L. }\end{array}$ & Acoraceae & & & \\
\hline \multirow[t]{3}{*}{54} & Cahong & $\begin{array}{l}\text { Piper nigrum } \\
\text { L. }\end{array}$ & Piperaceae & \multirow[t]{3}{*}{ Dizzy (cold) } & Dry fruit & \multirow[t]{3}{*}{$\begin{array}{l}\text { Pounded with } \\
\text { rice, smeared on } \\
\text { the forehead }\end{array}$} \\
\hline & Cekur & $\begin{array}{l}\text { Kaempferia } \\
\text { galanga L. }\end{array}$ & Zingiberaceae & & Rhizome & \\
\hline & Empuing & $\begin{array}{l}\text { Zingiber } \\
\text { purpureum }\end{array}$ & & & & \\
\hline
\end{tabular}




\begin{tabular}{|c|c|c|c|c|c|c|}
\hline & \multicolumn{3}{|c|}{ Roxb. } & & \multirow[b]{2}{*}{ Old Fruit } & \\
\hline & Pinong & $\begin{array}{l}\text { Areca } \\
\text { catechu L. }\end{array}$ & Arecaceae & & & \\
\hline 55 & Sorai & $\begin{array}{l}\text { Cymbopogo } \\
\text { n citratus } \\
\text { (DC.) Stapf }\end{array}$ & $\begin{array}{l}\text { Poaceae } \\
\text { (Gramineae) }\end{array}$ & Laryngitis & Stem & Burned, eaten \\
\hline \multirow[t]{4}{*}{56} & Empuing & $\begin{array}{l}\text { Zingiber } \\
\text { purpureum } \\
\text { Roxb. }\end{array}$ & Zingiberaceae & \multirow[t]{4}{*}{ Rheumatism } & Rhizome & $\begin{array}{l}\text { Pounded with } \\
\text { rice, smeared on } \\
\text { the part affected } \\
\text { by rheumatism }\end{array}$ \\
\hline & $\begin{array}{l}\text { Nangkok } \\
\text { belando }\end{array}$ & $\begin{array}{l}\text { Annona } \\
\text { muricata L. }\end{array}$ & Annonaceae & & Leaf & Boiled, drink \\
\hline & Putri malu & $\begin{array}{l}\text { Mimosa } \\
\text { pudica L. }\end{array}$ & Fabaceae & & $\begin{array}{l}\text { Leaf, } \\
\text { flower }\end{array}$ & \\
\hline & $\begin{array}{l}\text { Rumpot } \\
\text { sakong }\end{array}$ & \multicolumn{2}{|c|}{ Belum teridentifikasi } & & Leaf & $\begin{array}{l}\text { Pounded with } \\
\text { rice, smeared on } \\
\text { the part affected } \\
\text { by rheumatism }\end{array}$ \\
\hline 57 & Simpur & $\begin{array}{l}\text { Dillenia } \\
\text { suffruticosa } \\
\text { Griff. ex } \\
\text { Hook. }\end{array}$ & Dilleniaceae & Toothache & Leaf & $\begin{array}{l}\text { Pounded with } \\
\text { rice, pasted on } \\
\text { the tooth }\end{array}$ \\
\hline \multirow[t]{3}{*}{58} & Lilum & $\begin{array}{l}\text { Kalanchoe } \\
\text { pinnata } \\
\text { (Lam.) Pers. }\end{array}$ & Crassulaceae & \multirow[t]{3}{*}{ Headache } & \multirow[t]{2}{*}{ Leaf } & $\begin{array}{l}\text { Pounded with } \\
\text { rice, pasted on } \\
\text { forehead }\end{array}$ \\
\hline & $\begin{array}{l}\text { Pasok } \\
\text { matohari }\end{array}$ & $\begin{array}{l}\text { Clidemia } \\
\text { hirta(L.) } \\
\text { D.Don }\end{array}$ & $\begin{array}{l}\text { Melastomatacea } \\
e\end{array}$ & & & \multirow[t]{2}{*}{$\begin{array}{l}\text { Pounded, pasted } \\
\text { on the forehead }\end{array}$} \\
\hline & Rangki alus & $\begin{array}{l}\text { Capsicum } \\
\text { frutescens } \mathrm{L} .\end{array}$ & Solanaceae & & Ripe fruit & \\
\hline 59 & Bomban & $\begin{array}{l}\text { Donax } \\
\text { canniformis } \\
\text { (G.Forst.) } \\
\text { K.Schum. }\end{array}$ & Marantaceae & $\begin{array}{l}\text { Eye } \\
\text { pain/sore }\end{array}$ & $\begin{array}{l}\text { Shoots leaf } \\
\text { water }\end{array}$ & $\begin{array}{l}\text { Dripped into the } \\
\text { sore eye }\end{array}$ \\
\hline \multirow[t]{2}{*}{60} & Cekur & $\begin{array}{l}\text { Kaempferia } \\
\text { galanga L. }\end{array}$ & Zingiberaceae & \multirow[t]{2}{*}{ Stomachache } & \multirow[t]{2}{*}{ Rhizome } & $\begin{array}{l}\text { Pounded, } \\
\text { smeared on the } \\
\text { stomach }\end{array}$ \\
\hline & Empuing & $\begin{array}{l}\text { Zingiber } \\
\text { purpureum } \\
\text { Roxb. }\end{array}$ & & & & $\begin{array}{l}\text { Pounded with } \\
\text { rice, smeared on } \\
\text { the stomach }\end{array}$ \\
\hline \multirow[t]{2}{*}{$6 \mathrm{I}$} & Malai & $\begin{array}{l}\text { Benincasa } \\
\text { hispida } \\
\text { Cogn. }\end{array}$ & Cucurbitaceae & \multirow[t]{2}{*}{ Earache } & Old fruit & \multirow{2}{*}{$\begin{array}{l}\text { Pounded with } \\
\text { rice, smeared } \\
\text { behind the } \\
\text { earlobe }\end{array}$} \\
\hline & Pelok & $\begin{array}{l}\text { Manihot } \\
\text { esculenta } \\
\text { Crantz }\end{array}$ & Euphorbiaceae & & Tuber & \\
\hline
\end{tabular}




\begin{tabular}{|c|c|c|c|c|c|c|}
\hline 62 & Limau alus & $\begin{array}{l}\text { Citrus } \\
\text { aurantiifolia } \\
\text { Swingle }\end{array}$ & Rutaceae & Septic throat & Fruit & $\begin{array}{l}\text { Eaten right away } \\
\text { or make drinks }\end{array}$ \\
\hline & Sorai & $\begin{array}{l}\text { Cymbopogo } \\
n \text { citratus } \\
\text { (DC.) Stapf }\end{array}$ & $\begin{array}{l}\text { Poaceae } \\
\text { (Gramineae) }\end{array}$ & & Stem & Burned, eaten \\
\hline 63 & $\begin{array}{l}\text { Paku } \\
\text { enturuk alus }\end{array}$ & $\begin{array}{l}\text { Lygodium } \\
\text { flexuosum } \\
\text { (L.) Sw. }\end{array}$ & Schizaeaceae & Tuberculosis & Leaf & Boiled, drink \\
\hline 64 & Empuing & $\begin{array}{l}\text { Zingiber } \\
\text { purpureum } \\
\text { Roxb. }\end{array}$ & Zingiberaceae & Hemorroids & $\begin{array}{l}\text { Rhizome, } \\
\text { shoots }\end{array}$ & $\begin{array}{l}\text { Pounded with } \\
\text { rice, smeared on } \\
\text { the anus }\end{array}$ \\
\hline
\end{tabular}

